\title{
Social cognitive factors outweigh negative emotionality in predicting COVID-19 related safety behaviors
}

\author{
Grit Hein $^{\mathrm{a}, *, 1}$, Matthias Gamer ${ }^{\mathrm{b}, *, 1}$, Dominik Gall ${ }^{\mathrm{b}}$, Marthe Gründahl ${ }^{\mathrm{a}}$, Katharina Domschke ${ }^{\mathrm{c}, \mathrm{d}}$, \\ Marta Andreatta ${ }^{\mathrm{e}, \mathrm{b}}$, Matthias J. Wieser ${ }^{\mathrm{e}, \mathrm{b}}$, Paul Pauli ${ }^{\mathrm{b}}$ \\ ${ }^{a}$ Translational Social Neuroscience Unit, Department of Psychiatry, Psychosomatic and Psychotherapy, University of Würzburg, Germany \\ ${ }^{\mathrm{b}}$ Department of Psychology, University of Würzburg, Germany \\ ${ }^{\mathrm{c}}$ Department of Psychiatry and Psychotherapy, Medical Center - University of Freiburg, Faculty of Medicine, University of Freiburg, Freiburg, Germany \\ ${ }^{\mathrm{d}}$ Center for Basics in NeuroModulation, Faculty of Medicine, University of Freiburg, Freiburg, Germany \\ ${ }^{\mathrm{e}}$ Department of Psychology, Education and Child Studies, Erasmus University Rotterdam, The Netherlands
}

\section{A R T I C L E I N F O}

\section{Keywords:}

Social cognitive

Negative affect

Safety behavior

Survey

COVID-19

\begin{abstract}
A B S T R A C T
Emotion-motivation models propose that behaviors, including health behaviors, should be predicted by the same variables that also predict negative affect since emotional reactions should induce a motivation to avoid threatening situations. In contrast, social cognitive models propose that safety behaviors are predicted by a different set of variables that mainly reflect cognitive and socio-structural aspects. Here, we directly tested these opposing hypotheses in young adults $(N=4134)$ in the context of COVID-19-related safety behaviors to prevent infections. In each participant, we collected measures of negative affect as well as cognitive and socio-structural variables during the lockdown in the first infection wave in Germany. We found a negative effect of the pandemic on emotional responses. However, this was not the main predictor for young adults' willingness to comply with COVID-19-related safety measures. Instead, individual differences in compliance were mainly predicted by cognitive and socio-structural variables. These results were confirmed in an independent data set. This study shows that individuals scoring high on negative affect during the pandemic are not necessarily more likely to comply with safety regulations. Instead, political measures should focus on cognitive interventions and the societal relevance of the health issue. These findings provide important insights into the basis of health-related concerns and feelings as well as behavioral adaptations.
\end{abstract}

\section{Introduction}

According to influential emotion-motivation models (Bradley and Lang, 2007), behaviors are motivated by emotions. A situation or stimulus that is associated with positive emotions motivates approach behavior, i.e., behavior which maximizes the probability that the person will feel good again. In contrast, a situation or stimulus that is associated with negative emotions (e.g., fear) motivates avoidance behavior, i.e., behavior which maximizes the probability that the person will not experience these negative emotions again. Thus, emotion-motivation models predict that negative emotions result in avoidance behavior. In the context of the COVID-19 pandemic, avoidance behaviors are behaviors that comply with COVID-19-related safety regulations, because these behaviors reduce the probability to encounter threatening stimuli or situations such as COVID-19-related fear, depression, and traumatization (Cao et al., 2020), which have been shown to be related to individual differences in trait anxiety (Jungmann and Witthöft, 2020), uncertainty (Rettie and Daniels, 2020; Satici et al., 2020), and social factors (Qi et al., 2020). Based on emotion-motivation models, one would thus predict that COVID-19-related negative affect motivates avoidance of potential sources of infection, resulting in increased compliance with COVID-19-related safety behaviors. Importantly, emotion-motivation models do not account for the potential effect of socio-structural variables.

Other approaches have applied the core principles of social cognitive theory to health promotion and disease prevention (Bandura, 2004).

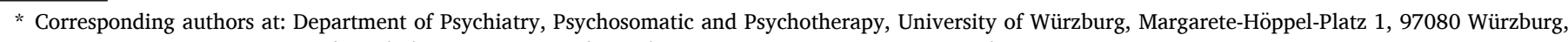
Germany (G. Hein). Department of Psychology, University of Würzburg, Marcusstr. 9-11, 97070 Würzburg, Germany (M. Gamer).

E-mail addresses: Hein_G@ukw.de (G. Hein), matthias.gamer@uni-wuerzburg.de (M. Gamer).

1 Equal contribution.
} 
According to social cognitive theory, effective health promotion requires a core set of individual and structural variables. Individual variables include knowledge of health risks and benefits, outcome expectations based on concerns regarding the severity and susceptibility to disease and its impact on everyday life routines, perceived self-efficacy that one can exercise control over one's health, the health goals people set for themselves and the concrete plans and strategies for realizing them. Structural variables are the perceived facilitators and social and structural impediments to changes the individual seeks, including media coverage. Importantly, social cognitive theory does not address emotional factors. Thus, predictors derived from the social cognitive theory are conceptually clearly distinct from the predictors proposed by emotion-motivation models (Bradley and Lang, 2007).

One recent study on an international sample has provided evidence for a link between outcome expectations, as derived from social cognitive models, and COVID-19-related safety behaviors (Clark et al., 2020). However, it did not address the relationship to negative affect. Other recent work that investigated the predictive effect of cognitive variables (knowledge about COVID-19) and affective variables (e.g., measures of negative emotionality) on COVID-19-related safety behavior in China highlighted the importance of affective variables (Shen et al., 2021), without addressing socio-structural factors. Thus, it remains elusive whether compliance with COVID-19-related safety behaviors is mainly driven by emotional variables as proposed by emotion-motivation models (Bradley and Lang, 2007) or by cognitive and socio-structural factors as proposed by social cognitive models of health behaviors (Bandura, 2004). Such differentiation is of immense importance because it could inform effective political measures to enhance safety behaviors.

The present cross-sectional study compared the evidence for emotional-motivational and social cognitive models predicting safety behavior based on survey data from a large group of young adults assessed during the first COVID-19-related lockdown in Germany. Enacted by the German government, citizens were instructed to only leave their homes for necessities like grocery shopping or doctor appointments, to work from home, and to minimize social contacts. Schools and universities were closed, as were buildings for religious ceremonies, the gastronomy, and facilities and institutions like playgrounds, gyms, theatres, cinemas, and museums.

According to emotion-motivation models, compliance with these COVID-19-related safety behaviors should be predicted by the same variables that also predict COVID-19-related negative affect. Alternatively, based on social cognitive models, compliance with safety behaviors should mainly be predicted by cognitive variables such as outcome expectations, socio-structural factors, and media influence, while emotions are expected to play a minor role.

\section{Method}

\subsection{Participants}

For the current study, we focused on a group of young adults since an infection with the SARS-Cov-2 virus in individuals below the age of 30 years is typically not associated with a severe course of the disease (Levin et al., 2020) but enhances the risk of spreading the virus to additional and potentially more vulnerable groups of the population. An adherence to safety behaviors is thus of crucial importance in young individuals. For participant recruitment, all students of three universities in Würzburg, Germany, were invited to complete an online questionnaire on a voluntary basis. Emails were sent to 26450 students of the Julius-Maximilians-University of Würzburg (58.8\% female), 8997 students of the University of Applied Sciences Würzburg-Schweinfurt ( $42.8 \%$ female), and 415 students of the University of Music Würzburg (51.8\% female). We furthermore asked colleagues to distribute the link to the online questionnaire to students of four additional universities in Germany (Humboldt-University of Berlin, Albert-Ludwigs-University of Freiburg, University of Hamburg, University of Münster) but in these cases no mass emails were sent out to potential participants and therefore only few students participated (see below). The study was approved by the ethics committees of the Department of Psychology, University of Würzburg, and of the Medical Faculty, University of Freiburg. All participants gave informed consent and did not receive compensation.

We collected a total of 5715 responses between April 8 and May 1, 2020. Completing the survey took, on average, $18.9 \mathrm{~min}(S D=5.8 \mathrm{~min})$. In a first step, we excluded data sets of participants who did not complete the entire survey (remaining $N=4373$ ). At the end of the questionnaire, we asked participants whether they gave valid answers to the questions. We excluded data sets from participants who negated this question (remaining $N=4355$ ). Furthermore, since the questionnaire did not require an access code, few people $(N=221)$ from outside the group of the seven previously mentioned German universities completed it. We decided to exclude these participants in order to have a relatively homogenous group of young adults (remaining and final $N=4134$ ). Most of these participants responded in the days from April 15 to April $22,2020(95.0 \%)$ and were students at one of the three universities in Würzburg, Germany (94.8\%) with response rates of $12.8 \%$ for the Julius-Maximilians-University of Würzburg, $5.0 \%$ for the University of Applied Sciences Würzburg-Schweinfurt, and $19.8 \%$ for the University of Music Würzburg. In the final sample, $67.5 \%$ of the participants were female, $31.3 \%$ male, $0.4 \%$ divers, and $0.8 \%$ did not disclose their gender. The mean age of the final sample was 22.3 years $(S D=4.4$ years); $7.2 \%$ of the participants did not disclose their age. Participants were mostly German citizens $(94.8 \% ; 1.0 \%$ did not disclose their citizenship).

\subsection{Measures}

The questionnaire consisted of proprietary questions about demographics, subjective assessments of the current situation, and participants' behavior as well as several standard questionnaires.

To address our main research question, we specified two relevant outcome variables: negative affect and compliance to safety behavior. Negative affect was estimated as the average of z-standardized scores of well-established questionnaires assessing state anxiety and depression (short-form of the state scale of the State-Trait Anxiety Inventory, STAIState (Marteau and Bekker, 1992); two-item version of the Patient Health Questionnaire, PHQ-2 (Löwe et al., 2005); and the Fear of COVID-19 Scale (Ahorsu et al., 2020)). Compliance to COVID-19-related safety behavior was calculated as the average of z-standardized ratings of the individual behavior in all important safety domains (i.e., the use of hand sanitizer, avoidance of touching potentially contaminated objects, keeping a safety distance towards other people, social drawback). Negative affect and compliance to safety behavior had a significant but relatively weak association, $r=0.18, p<.001$ (training data set, see description below).

Based on relevant models for predicting health-related behavior (Bandura, 2004; Bradley and Lang, 2007), the remaining questionnaire data could be separated into core variables related to social-cognitive models of health behavior (1. outcome expectations, 2. sociostructural factors, 3. media influence), core variables related to negative emotionality (4. trait anxiety and worry, 5. social factors, 6. uncertainty) as well as to general predictors (for a detailed description see Table S1). These categories encompassed questions on 1) the perceived risk that the COVID-19-pandemic affects different areas of individual health and wellbeing, susceptibility to the disease and fear of physiological symptoms. 2) Socio-structural factors included questions on perceived changes in research or health service priorities. 3) Media influence was assessed as duration and frequency of information exposure to COVID-19-related topics. 4) Trait anxiety and worry were measured using well-established trait questionnaires. 5) Social factors were covered by questions on social support and fear of loneliness but also included questions on ethnocentrism. 6) Uncertainty was assessed using a standard questionnaire on intolerance of uncertainty and a question on 


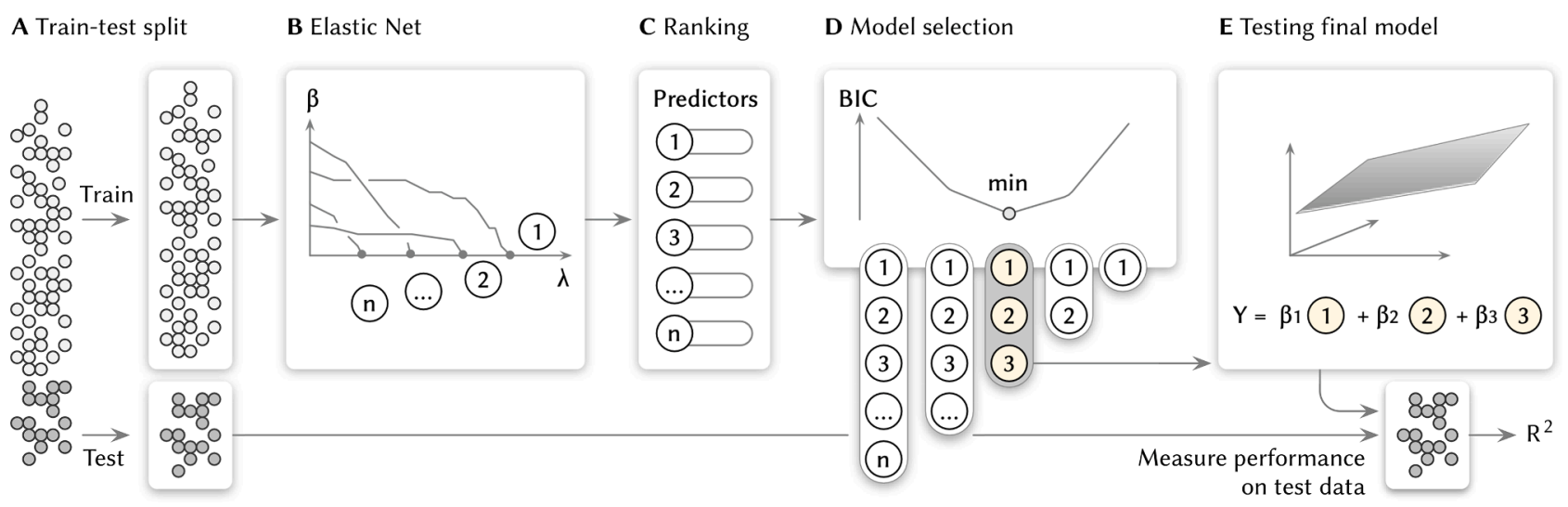

Fig. 1. Overview of analysis steps. A) The data set was randomly split into a training sample (80\%) and a test sample (20\%). Data of the training sample were used to establish models for predicting compliance with safety behaviors and negative affect (B) and to rank the candidate predictors (C). The Bayesian Information Criterion (BIC) was used to identify the set of predictors that contribute most to explaining compliance with safety behaviors and negative affect, i.e., result in the best model fit relative to model complexity (D). Finally, the generalizability of the model was tested on the independent test data set (E).

the individual certainty to meet the challenges imposed by the COVID19 pandemic. Regarding general predictors, we asked for gender, age, and housing situation as well as physical, work-related, and familyrelated activities (e.g., childcare). The list of variables is presented at https://osf.io/9xjgv/?view_only=a553c085109143338c1bdf5 $0907 a 1 b 05$.

\subsection{Data analysis}

Before data analysis, we randomly split the data into a training data set ( $80 \%, N=3307,67.6 \%$ female) and a test data set $(20 \%, N=827$, $67.5 \%$ female). We then used the training data set to build two predictive models, one for negative affect and one for compliance to safety behaviors (see Fig. 1 for an illustration of the procedure). Our analytic procedure was designed to determine which variables are most important for predicting these two outcome variables. Thus, in addition to selecting the most relevant variables (i.e., dimension reduction), we also aimed at determining their weights. In general, different statistical procedures have been proposed to address this issue with stepwise multiple regression models being the most parsimonious account. However, since we had no a priori knowledge on the covariance structure between potential predictors and since multiple regressions tend to yield unstable solutions in case of multicollinearity between predictors, we decided for using an alternative approach consisting of three steps.

1) Ranking of variables regarding their strength in predicting the outcome measures (Fig. 1B): For this purpose, we used Elastic Net regression which enables good prediction accuracy and interpretability of the statistical model in case of a high number of predictors with an unknown covariance structure (Zou and Hastie, 2005). It has the main advantage that it tends to balance the contribution of highly correlated predictors. In more detail, with increasing regularization, the Elastic Net tries to maximize the overall predictive performance of the model, independently of the beta coefficients of predictors in an unregularized model. As a result, Elastic Nets assign the highest rank to predictors that explain a unique portion of variance in the criterion (i.e., independently of other predictors), and predictors with large beta coefficients in an unregularized model can rank low due to their correlation with other predictors. Elastic Net regression therefore allows for selecting performant predictors from a large set of candidates and seems a suitable approach for dimension reduction in the current study. The models were built as follows: For each of the two dependent variables, we initially included all proprietary questionnaire items and questionnaire scores (z-standardized) in an Elastic Net regression (with an equal weight of the L1 and L2 penalty term). We then increased the regularization parameter lambda stepwise by 0.001 and recalculated the Elastic Net regression for each such lambda until the beta coefficients for all predictors equaled zero. This way, we ranked all predictors (Fig. 1C): the higher the lambda required to decrease the respective beta coefficient to zero, the higher the contribution of the predictor to the model's performance.

2) Identification of the most relevant set of predictors (Fig. 1D): Based on the ranking of predictors derived from the Elastic Net regression, we calculated a series of linear regression models by initially including all predictors and successively leaving out the predictor with the lowest rank, i.e., the smallest overall contribution. From these candidate models, we selected the one with the lowest Bayesian information criterion (BIC) value. The BIC considers the predictive value of the whole model and additionally incorporates a penalty for the number of predictors to reduce the risk of over-fitting the data. The finally selected model for each outcome measure therefore provides a good compromise between predictive strength and model complexity.

3) Testing model generalizability on an independent data set (Fig. 1E): After building the two models, we used the previously separated test data set to assess model generalizability. For this aim, we inserted the predictor values from the test data set into the selected model from step 2 . The beta-coefficients derived from the training data set hence defined the resulting predictions. We then compared the resulting predictions with the actual values of negative affect and safety behavior in the test data set. As an estimate of the models' performance, we used $R^{2}$ adjusted to quantify the correspondence between the resulting predictions and the actual values in the test data set.

For data processing and statistical analyses, we used Python 3.9.0 with the packages scikit-learn 0.23.2 (Pedregosa et al., 2012) and statsmodels 0.12.1 (Seabold and Perktold, 2010).

\section{Results}

The model explaining individual differences in compliance with safety behaviors was significant $(F(14,3293)=75.50, p<.001)$. It consisted of 14 predictors (selected via BIC) and explained about $24.0 \%$ of the variance ( $R^{2}$ adjusted) which is comparable to the prediction of health-related behaviors in other domains (McEachan et al., 2011). The top five predictors for compliance with COVID-19-related safety behaviors in young adults were related to outcome expectations (perceived susceptibility to disease in self and others), socio-structural factors emphasizing the societal relevance of the health issue (perceived 
A Compliance to safety behaviors Adj. $R^{2}=0.24$ (Training) 0.20 (Test)

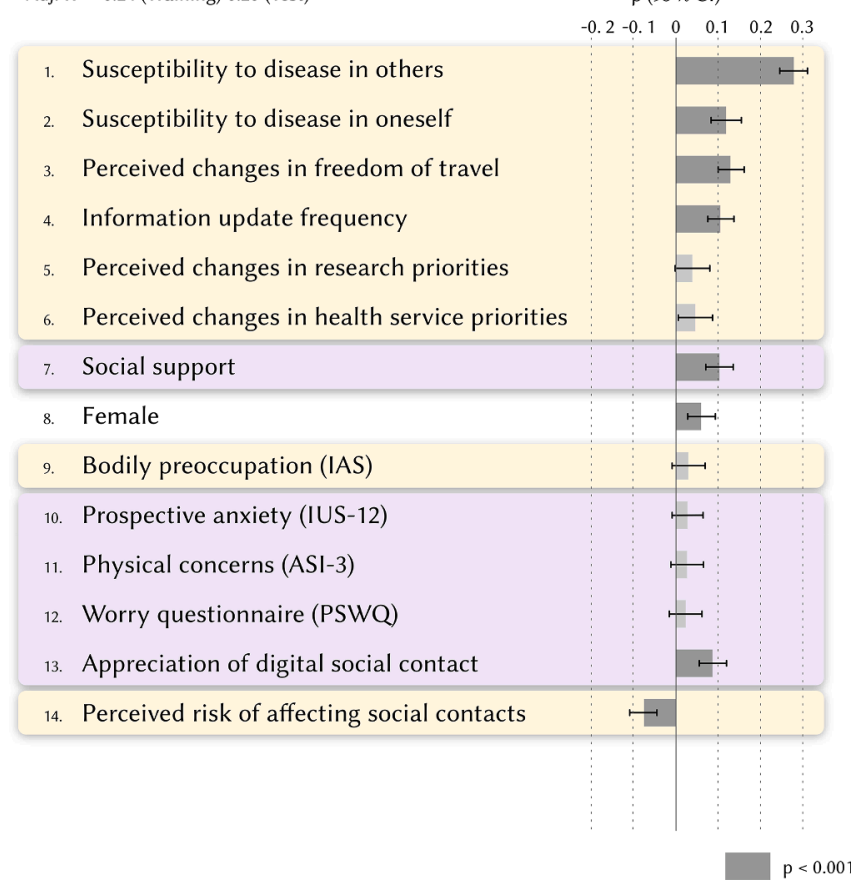

B Negative affect

Adj. $R^{2}=0.63$ (Training) 0.64 (Test)

$\beta(95 \% \mathrm{Cl})$

$\begin{array}{cccccc}-0.2 & -0.1 & 0 & 0.1 & 0.2 & 0.3\end{array}$

1. STAI trait

2. Fear of loneliness

3. Certainty to meet the challenge

4. Inhibitory anxiety (IUS-12)

5. Cognitive concerns (ASI-3)

6. Susceptibility to disease in oneself

7. Occupation/study concerns

8. Pleasant activities

9. Financial concerns

10. Unpleasant activities

11. Perceived risk of affecting cognitive performance

12. Prospective anxiety (IUS-12

13. Susceptibility to disease in others

14. Social support

15. Daily structure

16. Female

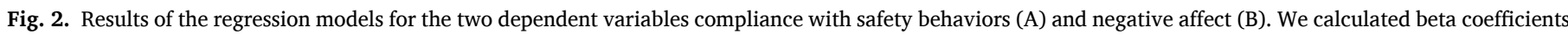

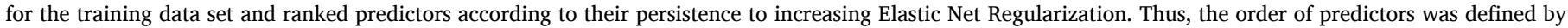

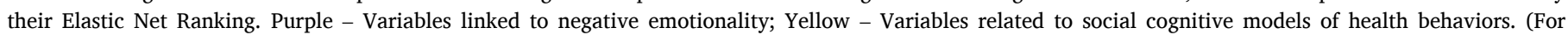
interpretation of the references to colour in this figure legend, the reader is referred to the web version of this article.)

changes in freedom of travel and research priorities), and media influence (individual differences in information uptake; Fig. 2A).

The model explaining individual differences in negative affect was also significant $(F(16,3291)=350.60, p<.001)$. It consisted of 16 predictors (selected via BIC) and accounted for $62.8 \%$ of variance. The strongest (top five) predictors of COVID-19-related negative affect in young adults were trait anxiety, social factors (fear of loneliness), and uncertainty (uncertainty to master the crisis; trait vulnerability to negative effects of uncertainty; Fig. 2B).

We were able to explain about $20.4 \%$ of the variance in safety behavior and about $64.1 \%$ of variance in negative affect when applying these models to the independent test data set. These results are mostly identical to the result obtained in the original analyses and thus confirm the predictor models' validity.

\section{Discussion}

Replicated in an independent test data set, our results show that social cognitive predictors outweigh the effect of negative emotionality on COVID-19-related safety behavior in young adults. We find that the pandemic in conjunction with the mandated containment measures indeed induced negative emotional reactions in young adults, as demonstrated by others (Gao et al., 2020; Giuntella et al., 2021; Qiu et al., 2020). For example, $28.4 \%$ of the current sample's participants reached scores $\geq 3$ in the PHQ-2, which has been suggested to indicate the presence of a depressive disorder (Löwe et al., 2005). Predictors of negative affect also played a role in predicting safety behavior, such as trait uncertainty, physical concerns and worries, which is in line with a recent study from China (Shen et al., 2021). In more detail, Shen and colleagues investigated the predictive effect of cognitive variables (knowledge about COVID-19) and affective variables (e.g., measures of negative emotionality) on COVID-19-related safety behavior. Their results showed that cognitive and affective variables significantly predicted safety behavior, with more variance explained by affective variables. Extending this previous work, our study investigated the effect of cognitive variables combined with socio-structural variables, as proposed by social cognitive theory (Bandura, 2004). According to our results, the top five predictors of COVID-19-related safety behaviors are a combination of socio-structural variables (e.g., perceived changes in freedom of travel; perceived changes in research priorities and health service priorities) and cognitive variables (perceived susceptibility to disease in self and others; information update frequency). These findings highlight the importance of socio-structural factors, combined with cognitive variables, and thus complement previous results that revealed an important role of affective variables (Shen et al., 2021).

According to our results, young adults who suffer most from negative emotions during the pandemic are not necessarily those who comply with COVID-19-related safety behaviors. Instead, compliance to safety behaviors was mainly predicted by outcome expectations and sociostructural factors emphasizing the societal relevance of the health issue. These findings support social cognitive models of health behavior (Bandura, 2004) and are in line with previous results from international investigations (Clark et al., 2020; Raude et al., 2020). Extending these previous findings, our study allows for a direct comparison of social cognitive health models to emotion-motivation models and shows that safety behaviors are not primarily driven by negative emotions that induce the motivation to avoid infections.

Our results are based on a relatively large sample, which, however, consisted of students, i.e., young adults with a high level of education. Since it is possible that the level of education mediates the predictive power of emotional and cognitive/ socio-structural factors on safety behaviors, respectively, it should directly be investigated in future studies whether the currently observed associations hold for people with lower levels of education.

The differences in model composition observed in the current study (Fig. 2) provide important insights into the basis of health-related concerns and feelings as well as behavioral adaptations during the COVID19 pandemic (Zvolensky et al., 2020) and can inspire models of health- 
related safety behavior. On the practical level, our results imply that compliance to safety behaviors in highly educated young adults might be improved by changing outcome expectations, for example by highlighting the severity and disruptiveness of the disease for the individual and close relatives as well as its societal relevance.

\section{Financial disclosures}

GH was supported by the German Research Foundation (HE 4566/51). PP, MJW, MA, KD and MGa were supported by the German Research Foundation (project no. 44541416, PP: B01 and Z02; MJW: B01; MA: B08; KD: C02 and Z02; MGa: C10). GH, MGa and PP were supported by the Volkswagenstiftung (Az 99451). The publication was supported by the Open Access Publication Fund of the University of Würzburg. The funding sources had no role in the design, conduct, or analysis of the study, or the decision to submit the manuscript for publication.

\section{CRediT authorship contribution statement}

Grit Hein: Conceptualization, Methodology, Validation, Writing original draft, Writing - review \& editing, Visualization, Supervision, Project administration. Matthias Gamer: Conceptualization, Methodology, Validation, Writing - original draft, Writing - review \& editing, Visualization, Supervision, Project administration. Dominik Gall: Conceptualization, Methodology, Validation, Software, Formal analysis, Investigation, Data curation, Visualization, Writing - review \& editing. Marthe Gründahl: Conceptualization, Methodology, Validation, Formal analysis, Investigation, Data curation, Visualization, Writing review \& editing. Katharina Domschke: Conceptualization, Resources, Writing - review \& editing. Marta Andreatta: Conceptualization, Resources, Writing - review \& editing. Matthias J. Wieser: Conceptualization, Resources, Writing - review \& editing. Paul Pauli: Conceptualization, Methodology, Validation, Writing - review \& editing, Supervision, Project administration.

\section{Declaration of Competing Interest}

The authors declare that they have no known competing financial interests or personal relationships that could have appeared to influence the work reported in this paper.

\section{Appendix A. Supplementary data}

Supplementary data to this article can be found online at https://doi. org/10.1016/j.pmedr.2021.101559.

\section{References}

Ahorsu, D.K., Lin, C.-Y., Imani, V., Saffari, M., Griffiths, M.D., Pakpour, A.H., 2020. The fear of COVID-19 scale: development and initial validation. Int. J. Ment. Health Addict. https://doi.org/10.1007/s11469-020-00270-8.

Bandura, A., 2004. Health promotion by social cognitive means. Heal. Educ. Behav. 31, 143-164. https://doi.org/10.1177/1090198104263660.
Bradley, M.M., Lang, P.J., 2007. Emotion and motivation, in: Cacioppo, J.T., Tassinary, L.G., Berntson, G. (Eds.), Handbook of Psychophysiology. Cambridge University Press, Cambridge, pp. 581-607. https://doi.org/10.1017/CBO9780511546396.025.

Cao, W., Fang, Z., Hou, G., Han, M., Xu, X., Dong, J., Zheng, J., 2020. The psychological impact of the COVID-19 epidemic on college students in China. Psychiatry Res. 287, 112934 https://doi.org/10.1016/j.psychres.2020.112934.

Clark, C., Davila, A., Regis, M., Kraus, S., 2020. Predictors of COVID-19 voluntary compliance behaviors: An international investigation. Glob. Transit. 2, 76-82. https://doi.org/10.1016/j.glt.2020.06.003.

Gao, J., Zheng, P., Jia, Y., Chen, H., Mao, Y., Chen, S., Wang, Y., Fu, H., Dai, J., 2020. Mental health problems and social media exposure during COVID-19 outbreak. PLoS One 15, 1-10. https://doi.org/10.1371/journal.pone.0231924.

Giuntella, O., Hyde, K., Saccardo, S., Sadoff, S., 2021. Lifestyle and mental health disruptions during COVID-19. Proc. Natl. Acad. Sci. USA 118, e2016632118. https:// doi.org/10.1073/pnas.2016632118.

Jungmann, S.M., Witthöft, M., 2020. Health anxiety, cyberchondria, and coping in the current COVID-19 pandemic: Which factors are related to coronavirus anxiety? J. Anxiety Disord. 73, 102239 https://doi.org/10.1016/j.janxdis.2020.102239.

Levin, A.T., Hanage, W.P., Owusu-Boaitey, N., Cochran, K.B., Walsh, S.P., MeyerowitzKatz, G., 2020. Assessing the age specificity of infection fatality rates for COVID-19: systematic review, meta-analysis, and public policy implications. Eur. J. Epidemiol. 35, 1123-1138. https://doi.org/10.1007/s10654-020-00698-1.

Löwe, B., Kroenke, K., Gräfe, K., 2005. Detecting and monitoring depression with a twoitem questionnaire (PHQ-2). J. Psychosom. Res. 58, 163-171. https://doi.org/ 10.1016/j.jpsychores.2004.09.006.

Marteau, T.M., Bekker, H., 1992. The development of a six-item short-form of the state scale of the Spielberger State-Trait Anxiety Inventory (STAI). Br. J. Clin. Psychol. 31, 301-306. https://doi.org/10.1111/j.2044-8260.1992.tb00997.x.

McEachan, R.R.C., Conner, M., Taylor, N.J., Lawton, R.J., 2011. Prospective prediction of health-related behaviours with the theory of planned behaviour: A meta-analysis. Health Psychol. Rev. 5, 97-144. https://doi.org/10.1080/17437199.2010.521684.

Pedregosa, F., Varoquaux, G., Gramfort, A., Michel, V., Thirion, B., Grisel, O., Blondel, M., Müller, A., Nothman, J., Louppe, G., Prettenhofer, P., Weiss, R., Dubourg, V., Vanderplas, J., Passos, A., Cournapeau, D., Brucher, M., Perrot, M., Duchesnay, É., 2012. Scikit-learn: machine learning in Python. J. Mach. Learn. Res. 39, i-ii.

Qi, M., Zhou, S.J., Guo, Z.C., Zhang, L.G., Min, H.J., Li, X.M., Chen, J.X., 2020. The effect of social support on mental health in Chinese adolescents during the outbreak of COVID-19. J. Adolesc. Heal. 67, 514-518. https://doi.org/10.1016/j. jadohealth.2020.07.001.

Qiu, J., Shen, B., Zhao, M., Wang, Z., Xie, B., Xu, Y., 2020. A nationwide survey of psychological distress among Chinese people in the COVID-19 epidemic: Implications and policy recommendations. Gen. Psychiatry 33, 1-4. https://doi.org/ 10.1136/gpsych-2020-100213.

Raude, J., Lecrique, J.M., Lasbeur, L., Leon, C., Guignard, R., du Roscoät, E., Arwidson, P., 2020. Determinants of preventive behaviors in response to the COVID19 pandemic in France: Comparing the sociocultural, psychosocial, and social cognitive explanations. Front. Psychol. 11, 1-15. https://doi.org/10.3389/ fpsyg.2020.584500.

Rettie, H., Daniels, J., 2020. Coping and tolerance of uncertainty: Predictors and mediators of mental health during the COVID-19 pandemic. Am. Psychol. 2 https:// doi.org/10.1037/amp0000710.

Satici, B., Saricali, M., Satici, S.A., Griffiths, M.D., 2020. Intolerance of uncertainty and mental wellbeing: Serial mediation by rumination and fear of COVID-19. Int. J. Ment. Health Addict. https://doi.org/10.1007/s11469-020-00305-0.

Seabold, S., Perktold, J., 2010. Statsmodels: econometric and statistical modeling with Python. Proc. 9th Python Sci. Conf. 92-96. https://doi.org/10.25080/majora92bf1922-011.

Shen, F., Min, C., Lu, Y., Chu, Y., 2021. The effect of cognition and affect on preventive behaviors during the COVID-19 pandemic: a cross-sectional study in China. BMC Public Health 21, 1-8. https://doi.org/10.1186/s12889-021-10784-y.

Zou, H., Hastie, T., 2005. Regularization and variable selection via the elastic net. J. R. Stat. Soc. Ser. B (Statistical Methodol.) 67, 301-320. https://doi.org/10.1111/ j.1467-9868.2005.00527.x.

Zvolensky, M.J., Garey, L., Rogers, A.H., Schmidt, N.B., Vujanovic, A.A., Storch, E.A., Buckner, J.D., Paulus, D.J., Alfano, C., Smits, J.A.J., O'Cleirigh, C., 2020. Psychological, addictive, and health behavior implications of the COVID-19 pandemic. Behav. Res. Ther. 134, 103715 https://doi.org/10.1016/j. brat.2020.103715. 